\title{
Research Paper \\ The Role of Health-promoting Lifestyle in Predicting Cognitive Status of Older Clergymen
}

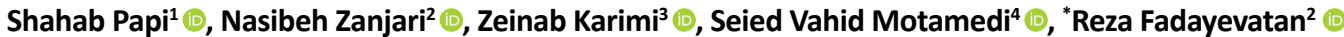

1. Department of Public Health, Social Determinant of Health Research Center, Faculty of Health, Ahvaz Jundishapur University of Medical Sciences, Ahvaz, Iran. 2. Department of Gerontology, Iranian Research Center of Aging, University of Social Welfare and Rehabilitation Sciences, Tehran, Iran.

3. Department of Health Education \& Promotion, School of Public Health, Qom University of Medical Sciences, Qom, Iran.

4. Representative of the Supreme Leader Institution, University of Social Welfare and Rehabilitation Sciences, Tehran, Iran.

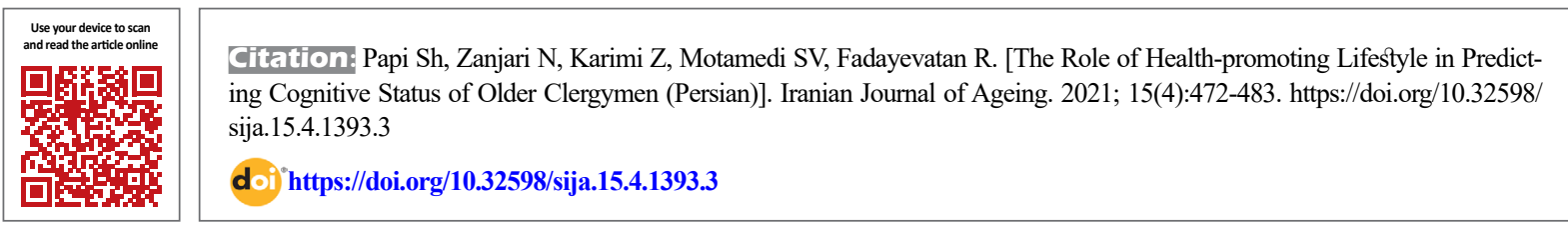

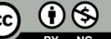

Received: 22 Dec 2019 Accepted: 22 Feb 2020 Available Online: 01 Jan 2021

Keywords: Health-promoting lifestyle, Cognitive status, Elderly, Clergy

\section{A B STRACT}

Objectives Healthy lifestyle along with spirituality affect the physical and mental health of older adults. Regarding the older clergymen's experience of spiritual life, this study aims to evaluate the role of healthpromoting lifestyle in predicting cognitive status of older clergymen.

Methods \& Materials This cross-sectional study was conducted on 190 older clergymen living in Qom, Iran who were selected using a convenience sampling method and based on the inclusion/exclusion criteria. Health Promoting Lifestyle Profile II and Mini-Mental State Exam were used to collect data. The collected data were analyzed in SPSS V. 22 software using descriptive and inferential statistics (Correlation test, linear regression analysis, independent t-test, and ANOVA). The significance level was set at 0.05.

Results The mean age of participants was $70.55 \pm 6.86$. There was a significant positive relationship between health-promoting lifestyle and cognitive status $(r=0.295, P=0.001)$. The results of multiple regression analysis showed that the dimensions of health responsibility and nutrition along with employment status explained almost $14 \%$ of the variance in cognitive status.

Conclusion it is necessary to pay more attention to nutrition and health responsibility in health-promoting interventions of older clergymen. Further studies with a qualitative approach are recommended to understand the mechanism of the effect of spirituality on health of older clergymen.

\section{Extended Abstract}

\section{Introduction}

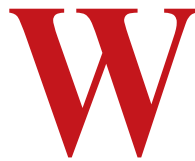

ith aging, the incidence of chronic diseases and their burden increase [1]. Cognitive disorders as a chronic disease is one of the most common psychological problems in the elderly [2]. Healthy lifestyles and healthpromoting behaviors can help maintain cognitive function, independence and psychological well-being, and reduce the incidence of chronic diseases in old age [3]. Spirituality is a powerful resource in late life providing the ability to adapt to individual needs and changes in old age [4]. Spirituality is an important factor in adapting to the consequences of aging and providing mental health to the elderly and is one of the dimensions of successful aging [5]. Given the importance of the role of religion in health, the question arises as whether older people who are more religious, have a better lifestyle and therefore better cognitive health? Identifying a health-promoting lifestyle influenced by religious teachings in this group can be effective and useful in improving the

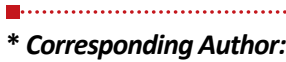

Address: Department of Gerontology, Iranian Research Center of Aging, University of Social Welfare and Rehabilitation Sciences, Tehran, Iran Tel: +98 (21) 2218004

E-mail: reza1092@yahoo.com 
health behaviors of other elderly groups in religious communities. Therefore, considering the importance of healthpromoting lifestyle in the prevention or control of mental disorders in old age and due to the lack of studies in the field of health-promoting lifestyle of the religious elderly, this study aims to determine the effect of health-promoting lifestyle on the cognitive status of older clergymen in Iran.

\section{Methods}

This is a cross-sectional study conducted on older clergymen in Qom, Iran in 2018. The sample size was obtained 176 using the formula based on correlation coefficient of $r=$ 0.23 in Lee et al.'s study [6], 95\% test power, and 5\% error. Assuming a 15\% drop in individuals, final sample size was set as 190. Participants were selected using a convenience sampling method. Age $\geq 60$ years, ability to communicate and willingness to participate in the study were the inclusion criteria. Those who had no willingness to continue participation were excluded from the study. In order to collect information, Health-Promoting Lifestyle Profile II (HPLP-II), Mini-Mental Status Examination (MMSE) and a checklist of demographic and disease information were used. Data were collected in SPSS V. 22 software using descriptive statistics (mean, standard deviation, and frequency) and inferential statistics (independent t-test, ANOVA, correlation test, and multiple linear regression analysis). The significance level in the tests was considered 0.05 .

\section{Results}

The mean age of participants was $70.55 \pm 6.86$ years. They had an income $>1$ million Tomans per month. Results showed their higher mean HPLP-II scores $(\mathrm{t}=5.07$; $\mathrm{P}=$ 0.001). The results of t-test showed that the mean score of MMSE was significantly different among the job groups where the subjects who had a full-time job had a higher score than those with part-time job $(\mathrm{t}=2.4 ; \mathrm{P}=0.01)$. There HPLP-II dimensions of health responsibility, physical activity, nutrition, stress management and overall HPLP-II score had a positive and significant correlation with cognitive status in the elderly $(\mathrm{P}=0.001 ; \mathrm{r}=0.295)$ (Table 1$)$. In multiple regression analysis, the dimensions of health responsibility and nutrition along with employment status explained about $14 \%$ of the variance in cognitive status (Table 2).

\section{Conclusion}

Health responsibility and nutrition were the predictors of cognitive status in older clergymen. Studies have shown that health and quality of life are important indicators of psychological well-being [7]; as a result, by improving the quality of life and promoting healthy behaviors, the psychological well-being of the elderly increases. On the other hand, Baruth et al. showed high healthy behaviors of African-American pastors [8]. This finding can be a reason for the impact of religious teachings and spirituality on health responsibility and attention to have a health-promoting lifestyle in the elderly. Mahmoudi et al. showed the effect of spiritual health on the adoption of self-care behaviors in the elderly [9]. Some studies have examined the relationship between religion and the dietary regime of the elderly and have reported religion as one of the influential factors in the nutrition of the elderly [10].

The elderly clergymen in our study had no cognitive impairment. Due to the fact that the clergymen are educated groups due to their jobs and constantly involving in topics such as philosophy and jurisprudence and have higher skills in recording and remembering events and in other cognitive cases by maintaining mental functional abilities, they are not exposed to risk factors that can cause cogni-

Table 1. Test results of examining the correlation between health-promoting lifestyle and cognitive status in the elderly

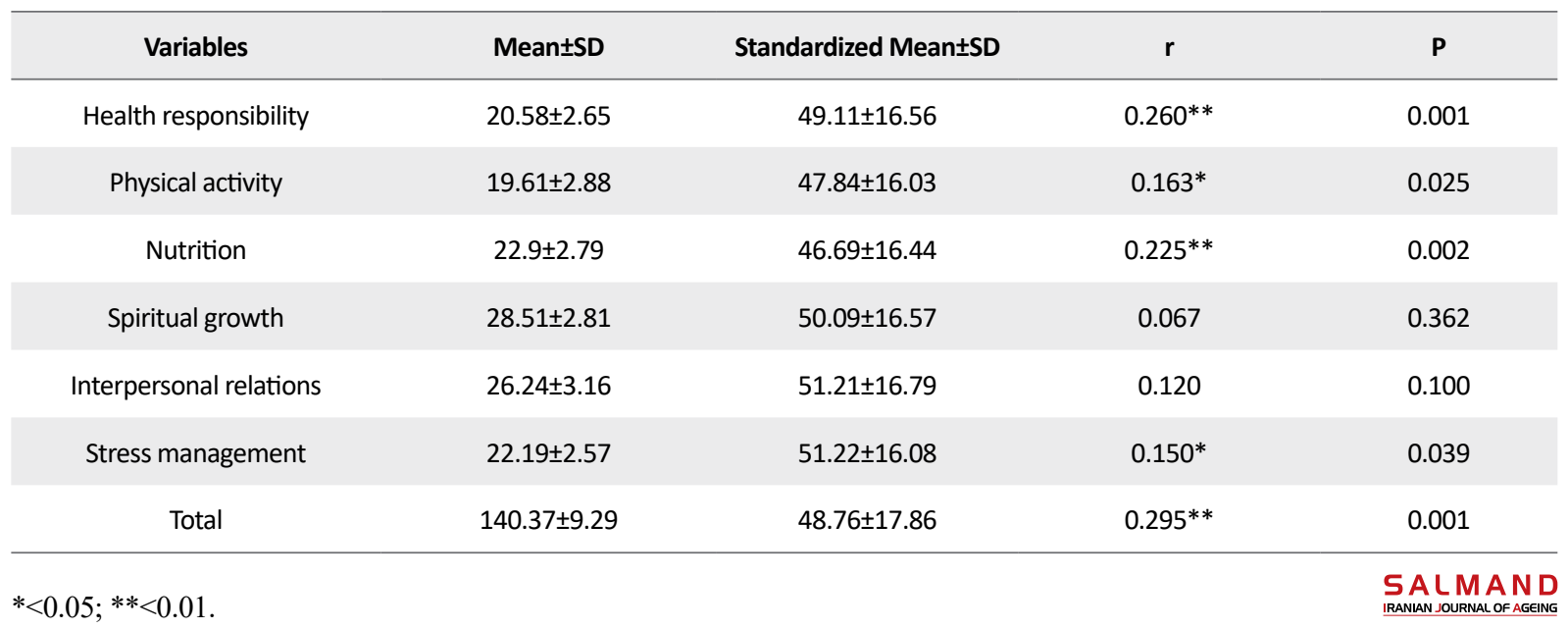


Table 2. Multiple linear regression coefficients of the relationship between HPLP-II dimensions and cognitive status

\begin{tabular}{|c|c|c|c|c|}
\hline \multirow{2}{*}{ Predictor } & \multicolumn{2}{|c|}{ Model 1} & \multicolumn{2}{|c|}{ Model 2} \\
\hline & Beta & $\mathbf{P}$ & Beta & $\mathbf{P}$ \\
\hline Health responsibility & 0.215 & 0.003 & 0.194 & 0.008 \\
\hline Physical activity & 0.128 & 0.084 & 0.120 & 0.101 \\
\hline Nutrition & 0.170 & 0.026 & 0.170 & 0.025 \\
\hline Spiritual growth & -0.015 & 0.832 & -0.031 & 0.673 \\
\hline Interpersonal relations & 0.075 & 0.300 & 0.085 & 0.236 \\
\hline Stress management & -0.016 & 0.847 & -0.011 & 0.892 \\
\hline Employment status (full-time) & & & -0.143 & 0.043 \\
\hline F (sig.) & \multicolumn{2}{|c|}{$4.190(0.001)$} & \multicolumn{2}{|c|}{$4.247(0.001)$} \\
\hline$R\left(R^{2}\right)$ & \multicolumn{2}{|c|}{0.348 (12\%) } & \multicolumn{2}{|c|}{0.37 (14\%) } \\
\hline
\end{tabular}

tive impairment [11]. According to the results of this study, more attention should be paid to the dimensions of nutrition and health responsibility in interventions for promoting the healthy behaviors of the elderly.

\section{Ethical Considerations}

Compliance with ethical guidelines

This study was approved by Ethical Coommittee of University of Social Welfare and Rehabilitation Sciences (Code: IR.USWR.REC.1397.49). All ethical principles are considered in this article. The participants were informed about the purpose of the research and its implementation stages. They were also assured about the confidentiality of their information and were free to leave the study whenever they wished, and if desired, the research results would be available to them.

Funding

This study was supported by the Deputy for Research of the University of Social Welfare and Rehabilitation Sciences (Grant No.: 2004)

Authors' contributions

Conceptualization: Shahab Papi, Reza Fadayevatan; Investigation: Shahab Papi, Nasibeh Zanjari, Zeinab karimi; Editing \& review: Nasibeh Zanjari, Zeinab karimi; Project administration: Reza Fadayevatan.

\section{Conflicts of interest}

The authors declare no conflict of interest.

\section{Acknowledgements}

The authors would like to thank the Vice-Chancellor for Research of the University of Social Welfare and Rehabilitation Sciences and all participants for their cooperation. 


\title{
نقش ييشبينى كثنده سبك زندكى ارتقادهنده سلامت در وضعيت شناختى سالمندان مرد روحانى
}

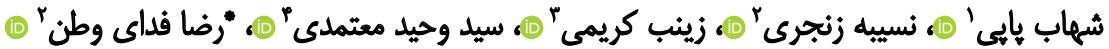

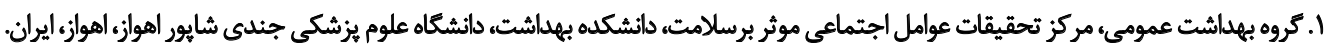

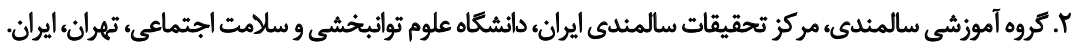

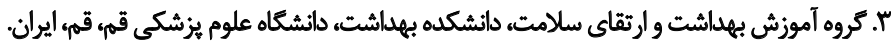

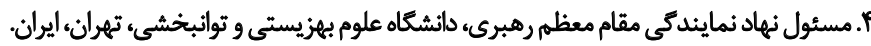

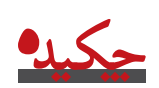

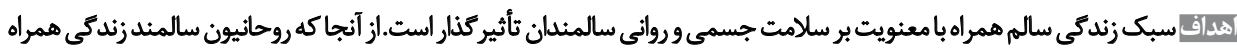

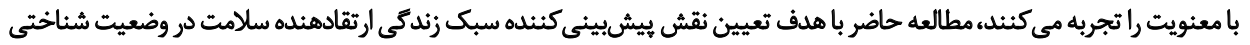

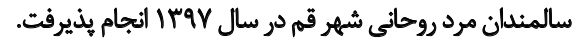

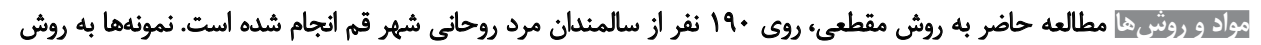

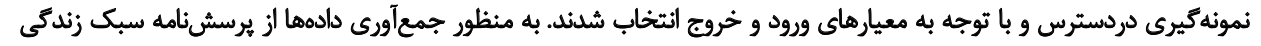

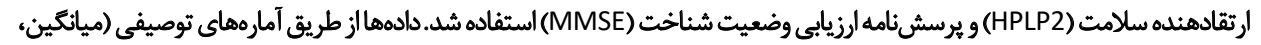

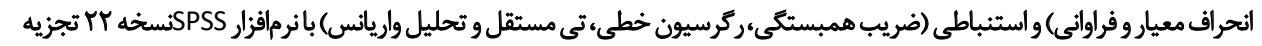

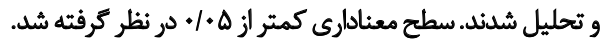

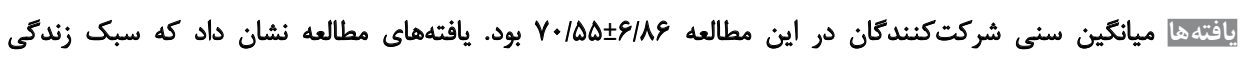

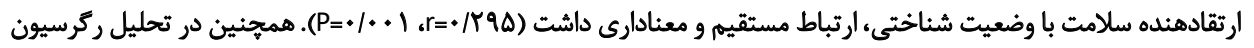

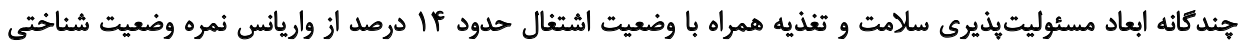

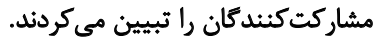

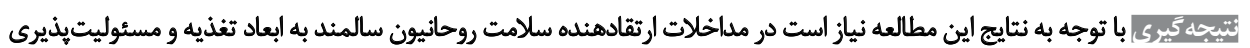

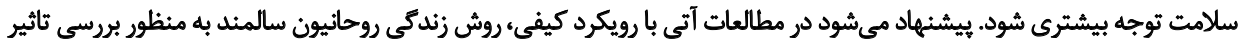

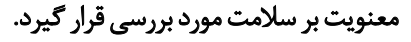

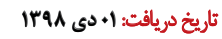
تاريخ بذيرش: r. ت اسفند

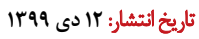

ارتباط هستند، اشاره كرد [Q]]. از سوى ديكر با افزايش سن ميزان

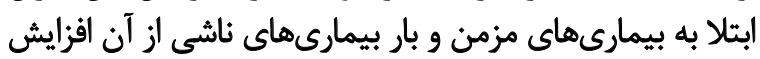

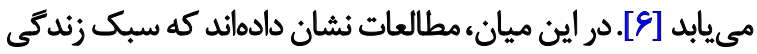

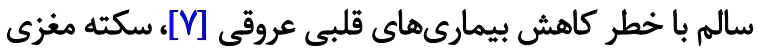

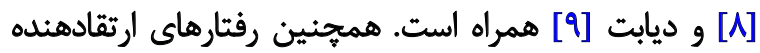

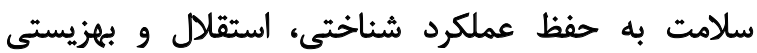
روانشناختى در دوران سالمندى كمك ميكى كند [• [1].

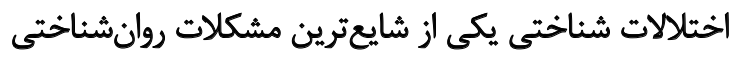

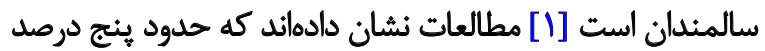

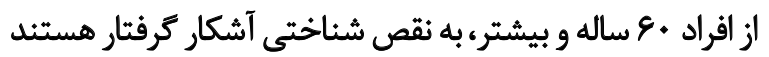

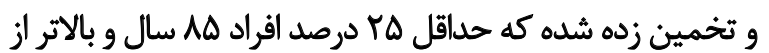

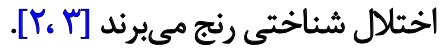

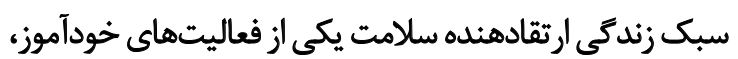

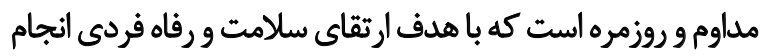

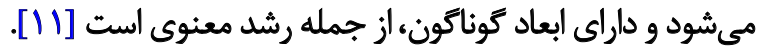

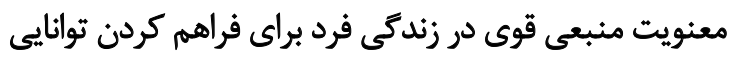

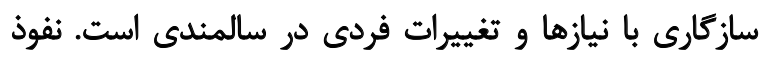


نمونه از روش نمونه ئيرى در دسترس استفاده شد. حجم نمونه

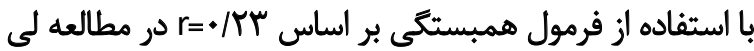

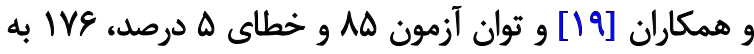

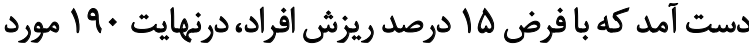

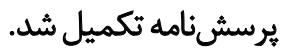

نمونهها به روش نمونه تيرى در دسترس و با توجه به معيارهاي

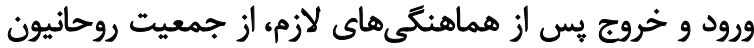

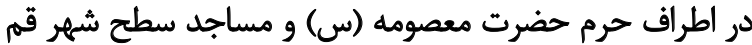

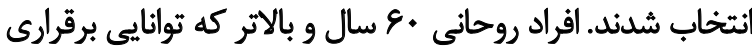

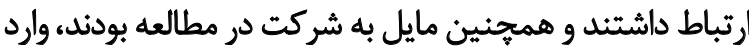

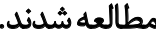

روحانيون سالمندى كه هنكام مطالعه تمايلى به ادامه مشاركت

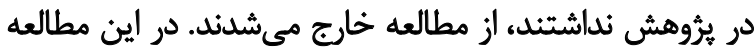

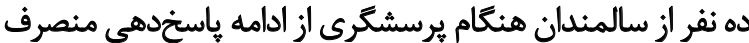

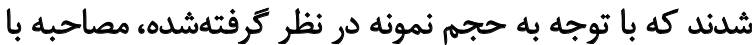

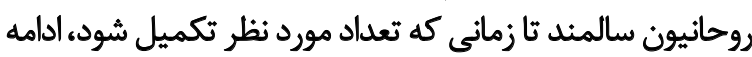

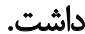

يرسشنامهها توسط يرسشكر آموزشديده تكميل شد. به

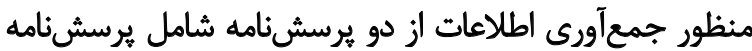

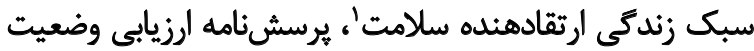

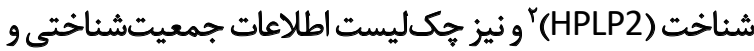
بررسى فراوانى بيمارىها در سالمندان استفاده شُد.

يرسش نامه سبك زئدكى سالم از ها هو سؤال تشكيل شده كه

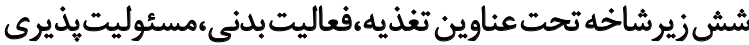

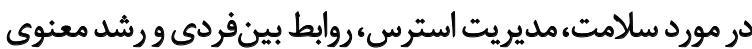

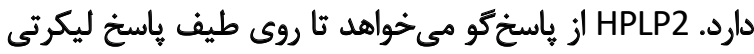

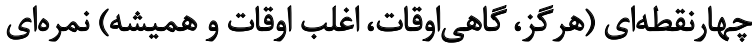
بين يك ثا جهار را به خود اختصاص دهاهد

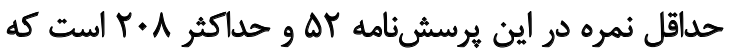

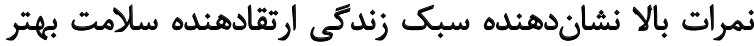

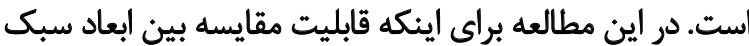

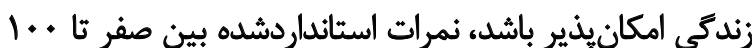
نيز ارائه شده است.

اين يرسشنامه توسط والكر و همكاران در سال 199 199 ساخته

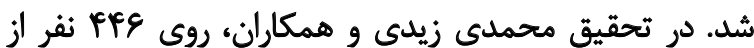

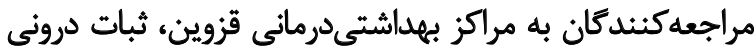

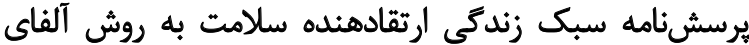

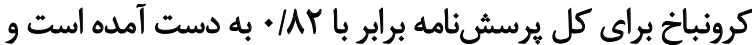

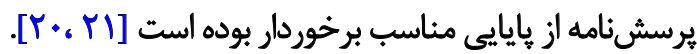

1. Health-Promoting Lifestyle Profile II (HPLP2)

2. Mini-Mental Status Examination (MMSE)
معنويت در ابعاد جسمانى زندكى انسانها تأثير بسزايي در ارتباط

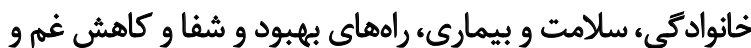

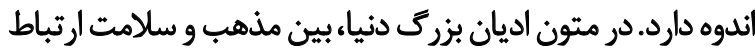

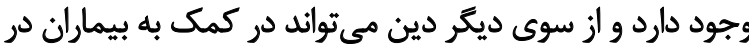

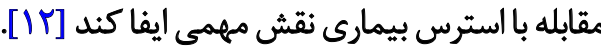

اعتقادات و عملكردهاى دينى عامل مهيمى در سازگكارى با

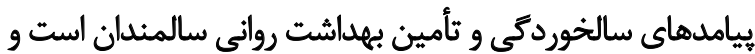

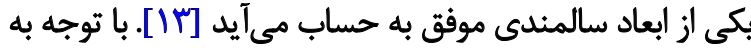

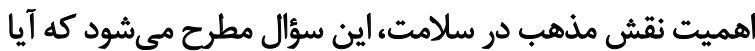

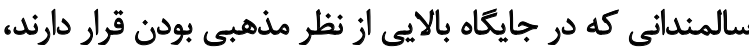

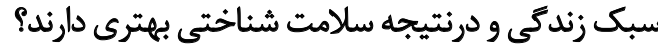
مطالعات اوليه كه روى طول عمر تمركز دارند، نشان مي دهند

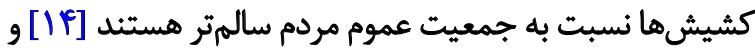

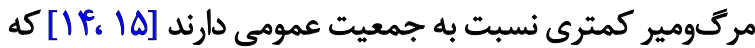

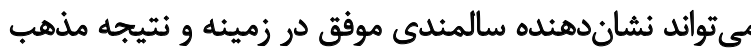

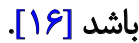

بسيارى از مطالعات در ارتباط با نقش روحانيت و يا آموزههاى

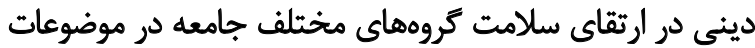

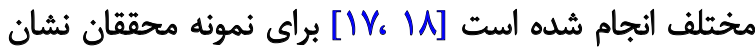

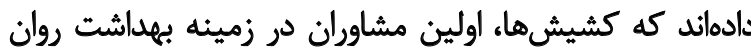

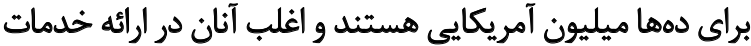

بهداشت روان با متخصصين بهداشت همكارى دارند [1/] با اين حال، مطالعهاى كه به بررسى سبك زندكى و وضعيت

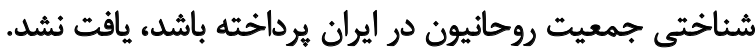

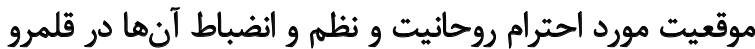

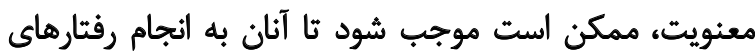

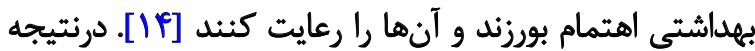

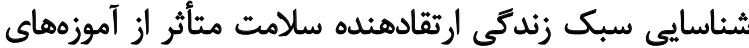

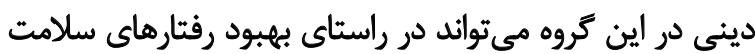
ساير كروههاى سالمندى در جوامع مذهبى مؤثر و مفيد باشد دئ. از اين رو با توجه به اهميت سبك زندكى ارتقادهنده سلامت

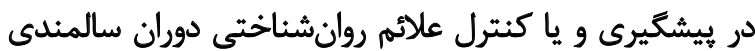

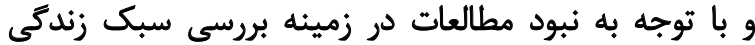

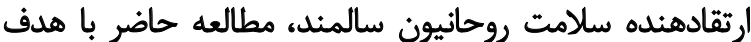

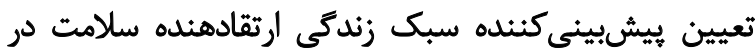

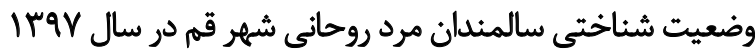

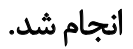

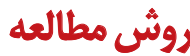

مطالعه حاضر يكى مطالعه مقطعى است كه در ميان روحانيون

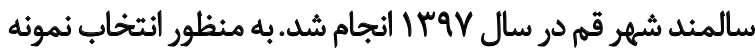

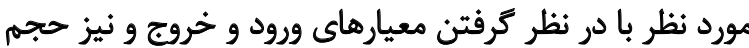




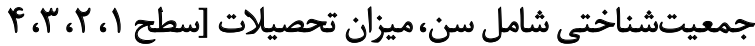

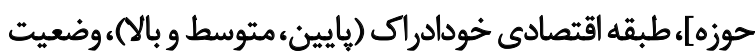

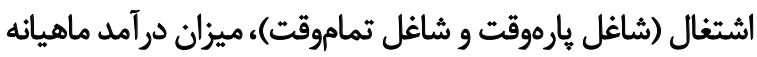

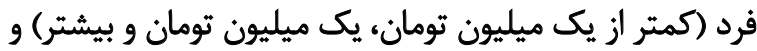
وضعيت مسكن (ملكى و اجارهاى) استفاده شد.

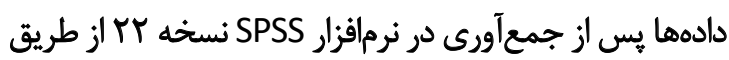

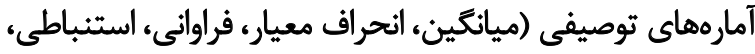

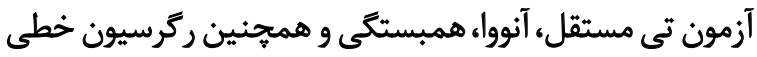

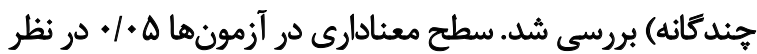

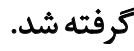

يافتهها

بر اساس يافتههاى مطالعه ميانگين سنى جمعيت تحت

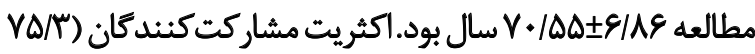
درصد) شاغل تماموقت بودند و طبقال را متوسط (1T/1 درصد) ذكر كردند. بيشتر روحانيون سالمثلد
در مطالعه حاضر نيز ميزان آلفاي كرونباخ بام درصد بود. جهت

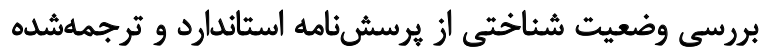
(باري (MSE)

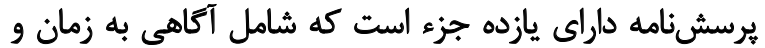

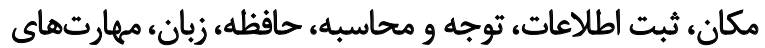

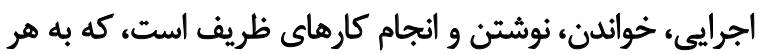
هاسخ صحيح يك امتياز تعلق مى نيردان درمجموع حداكثر نمره كسبشده · بامتياز در نظر كرفته شده

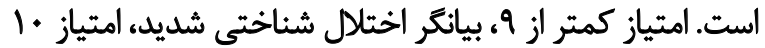

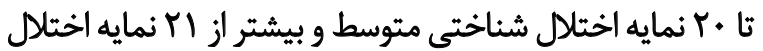

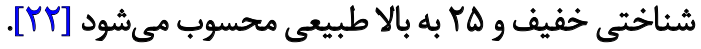
روايى و يايايي آن توسط فروغان و همكاران روى • rV سالمند

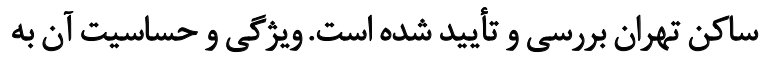

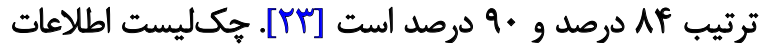

3. Mini-Mental Status Examination

جدول ا. ارتباط بين وضعيت سبك زئدكى ارتقادهنده سلامت و وضعيت شناختى با متغيرهاى جمعيتشناختى در ميان روحانيون سالمند

\begin{tabular}{|c|c|c|c|c|c|c|c|c|}
\hline \multirow[b]{2}{*}{$\mathbf{P}$} & \multicolumn{2}{|c|}{ وضعيت شيناختي } & \multicolumn{3}{|c|}{ سبك زندكي ارتقادهنده سلامت } & \multirow{2}{*}{ (درواوانى } & \multirow{2}{*}{\multicolumn{2}{|c|}{ متغير هاى جمعيتششناختى }} \\
\hline & آمارئ آزون & انحراف معيار & $\mathbf{P}$ & آزاريون & انحراف معيار & & & \\
\hline \multirow{3}{*}{$. / N \mid$} & \multirow{3}{*}{$\mathrm{F}=\cdot / A$} & $r V / A V \pm \cdot / \Delta P$ & \multirow{3}{*}{ love } & \multirow{3}{*}{$\mathrm{F}=\cdot / \Delta \Delta$} & $\| f \cdot / H T \pm q / I V$ & IFr(VT/V) & Vo- 8. & \multirow{3}{*}{ كروهناي سنى } \\
\hline & & $r q / A F \pm \cdot / \Delta r$ & & & IF·/Ar土NVq & $F \Delta(\pi T / V)$ & $1 \Delta-V \Delta$ & \\
\hline & & $r . \pm .1 . .1$ & & & $1 r \Delta \pm F / T M A$ & $r(V / 8)$ & +10 & \\
\hline \multirow{3}{*}{. NPr } & \multirow{3}{*}{$F=* / 1 f$} & $r \sqrt{ } / \Delta A \pm / P r$ & \multirow{3}{*}{ - I8AV } & \multirow{3}{*}{$F=\cdot / r V$} & $|r q / 4| \pm q / q Q V$ & $\operatorname{Ir}(\mathscr{R / M})$ & سطح r & \multirow{3}{*}{ تحصيلات } \\
\hline & & $r \vee / \wedge q \pm . / r r$ & & & $\mid f \cdot / \cdot Y \pm V \Delta A$ & $119(8 \times / 9)$ & سطح r & \\
\hline & & $r q / A \pm \pm .1 .8$ & & & $|F| / T \cdot \mid \pm N E E$ & $\Delta q\left({ }^{N} 1 / 1\right)$ & سطح F & \\
\hline \multirow{2}{*}{$\circ / \cdot 14$} & \multirow{2}{*}{$t=Y / P Q q$} & & \multirow{2}{*}{.$/ 10 T^{e}$} & \multirow{2}{*}{$\mathrm{t}=1 / \mathrm{F}^{\mathrm{c}}$} & & & شاغل تماموقت & \multirow{2}{*}{ وضعيت أشتغال } \\
\hline & & $r q / \cdot Y \pm \cdot / 9 \Delta$ & & & ITNFA土NQqT & Pr(TEM & شاغل يارهوقت & \\
\hline \multirow{2}{*}{$\cdot I \Delta Q A$} & \multirow{2}{*}{$t=-\cdot / \Delta A$} & $r q / A r \pm+(\Delta)$ & \multirow{2}{*}{$.1 . .1$} & \multirow{2}{*}{$t=-\bowtie / \cdot r$} & $\mid r E / q \& \pm N \& q$ & $q+(f V / 4)$ & كمتر از يك ميليون تومان & \multirow{2}{*}{ فر آمد ماهيانه } \\
\hline & & $r q / \wedge q \pm \cdot / \Delta f$ & & & $|F \Psi / F| \pm N V E$ & $1+\circ(\Delta r / g)$ & يك ميليون تومان و بيشتر & \\
\hline \multirow{2}{*}{ 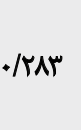 } & \multirow{2}{*}{$t=-v / \cdot v$} & $r q / V A \pm \cdot / A \Delta$ & \multirow{2}{*}{. life } & \multirow{2}{*}{$\mathrm{t}=1 / 7 \Delta$} & IFT/THEI+/AA & $\mathbb{T}(19 / \Delta)$ & 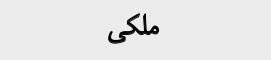 & \multirow{2}{*}{ وضعيث مسكن } \\
\hline & & $r q / M \pm \cdot / R T r$ & & & $\| r q / A V \pm N A r$ & $1 \Delta r(\lambda \cdot / \Delta)$ & الجارواي & \\
\hline \multirow{3}{*}{ + NEV } & \multirow{3}{*}{$\mathrm{F}=1 / f 8$} & $r q / V \pm V / * r^{\circ}$ & & & $179 / 9 \Delta \pm N A M$ & $M e(M r / P)$ & بايين & \multirow{3}{*}{ طبقه |قتصادى } \\
\hline & & $r q / M \pm \cdot / R r$ &.$/ N E Y$ & $\mathrm{~F}=\cdot / \mathrm{R}$ & 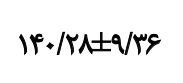 & $10 \&(A T / M)$ & هتوسط & \\
\hline & & $r+ \pm+1++1$ & & & $\mid r T / F \pm 1 \cdot / r \lambda$ & $1 \cdot(\Delta / \pi)$ & بالا & \\
\hline
\end{tabular}

ك 
جدول r. ضرايب همبستكى بين سبك زندكى ارتقادهنده سلامت و ابعاد آن با وضعيت شناختى روحانيون سالمند

\begin{tabular}{|c|c|c|c|c|}
\hline $\mathbf{P}$ & ضريب همبستكى & ميانكين استاندار رشدهد|انحراف معيار & ميانكين +انحراف معيار & مثغير \\
\hline$+1+1$ & 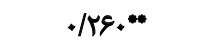 & $P q / 11 \pm 18 / \Delta S$ & $r+/ \Delta \Lambda \pm Y / \varepsilon \Delta$ & مسئوليتيذيرى سلامت \\
\hline $.1 \cdot \mathrm{TA}^{2}$ &.$/ 19 \%$ & PV/AF $\pm \mid \& / \cdot Y$ & $\mid V / E I \pm T / M$ & فعاليت فيزيكى \\
\hline $.1 .0 r$ & . /MrO** & $p q / 9 q \pm 18 / p 4$ & $r r / q \pm r / v q$ & تغذيه \\
\hline . & $.1 .8 \mathrm{~V}$ & $\Delta .1 .9 \pm 18 / \Delta \mathrm{V}$ & $r N \Delta) \pm Y(A)$ & رشد معنوى \\
\hline .11. &.$/ 17$ & $\Delta / / T \backslash \pm 18 / N q$ & $r e / r e \pm m / l e$ & روابط يينفردى \\
\hline .1 .19 &.$/ 1 \theta^{* *}$ & $\Delta V / T Y \pm \mid \varnothing / \cdot \Lambda$ & $r T / 19 \pm Y / \Delta Y$ & مديريت استرس \\
\hline .1 .01 &.$/ 179 \Delta^{* * *}$ & PNVE \pm IVIAE & $18 \cdot / T V \pm q / T q$ & سبك زندكى كل \\
\hline
\end{tabular}

در بررسي وضعيت شناختي نيز نتايج آزمون تي تست نشان

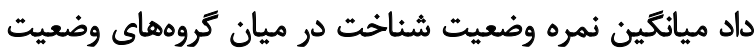

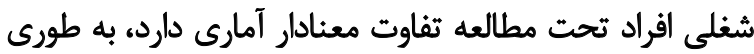

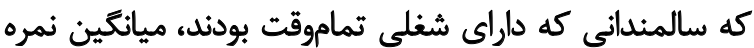

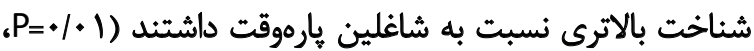

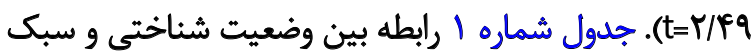

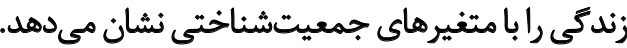

بر اساس يافتههاي هطالعه، ميانئين استاندارد نمره سبك

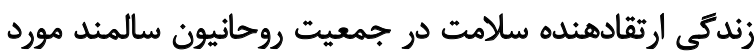
مطالعه FN IV VE

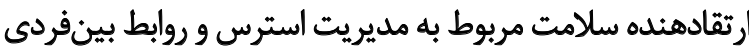

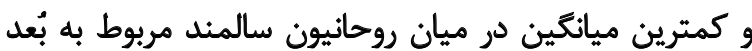

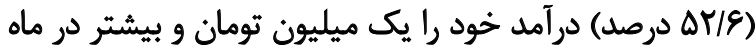

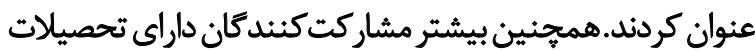

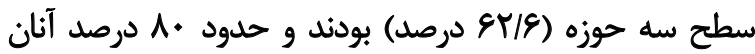
مسكن اجارهاى داشئند.

همان كونه كه در جدول شماره ا 1 مشاهده ميشود، نتايج

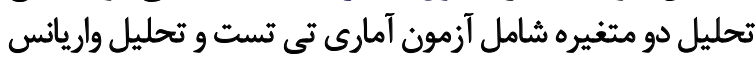

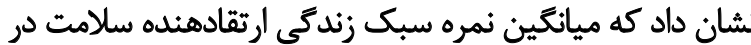

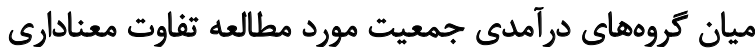

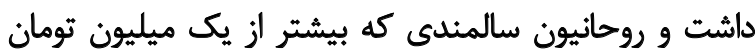

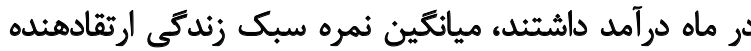

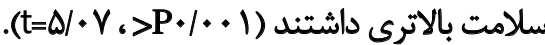

جدول با. ركرسيون خطى جَندكائه رابطه ابعاد سبك زندكى با نمره وضعيت شناختى با / بدون كنترل متغير جمعيتشئاختى

\begin{tabular}{|c|c|c|c|c|}
\hline \multicolumn{2}{|c|}{ مدل دوم } & \multicolumn{2}{|c|}{ 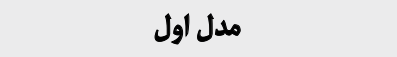 } & \multirow{2}{*}{ مثغير ييشبين } \\
\hline $\mathbf{P}$ & Beta & $\mathbf{P}$ & Beta & \\
\hline$+1+1$ &.$/ 194$ & r. & . Mia & مستوليتئيرى سلامت \\
\hline $.11+1$ &.$/ N r$ & $+1+A F$ & ./YA & فعاليت فيزيكى \\
\hline $.1 \cdot T \Delta$ &.$/ 1 \mathrm{r}$ & .1 .48 &.$/ \mathrm{V}$ & تفذيه \\
\hline $.18 n$ &.$- \mid \cdot m$ & . AMT & $-.1 \cdot 10$ & رشد رعنوى \\
\hline - ( & $\cdot 1 \cdot 10$ &.$/ \%$ & $.1 \cdot V a$ & روابط بينفردى \\
\hline -/A9r &.- .1 .11 & - /APr & $-.1 \cdot 18$ & مديريت استرس \\
\hline $1 \cdot \pi$ &.$- / 1 P r$ & & & وضعيت اششتال (تماموقت ه ) \\
\hline \multicolumn{2}{|c|}{$\operatorname{PRPV}(\cdot 1 \cdot \cdot 1)$} & \multicolumn{2}{|c|}{$p / 19 \cdot(\cdot 1+.1)$} & F model ( $p$-value) \\
\hline \multicolumn{2}{|c|}{.$/ T V\left(\% \mathbb{N}^{p}\right)$} & \multicolumn{2}{|c|}{.$/ \operatorname{men}(\% 1 \mathrm{~T})$} & $\mathbf{R}\left(\mathbf{R}^{\top}\right)$ \\
\hline
\end{tabular}




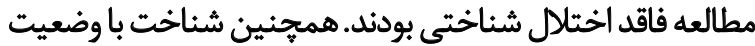

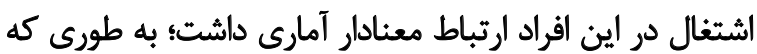

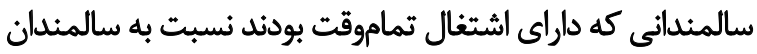

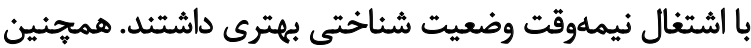

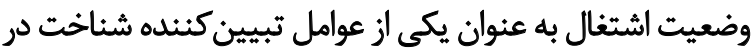

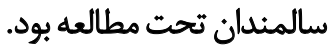

در تبيين اين موضوع مي توان به اين نكته اشاره كرد كه با توجه

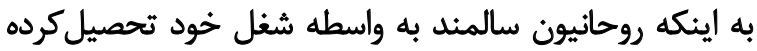

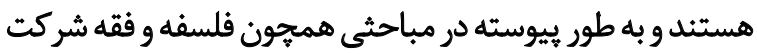

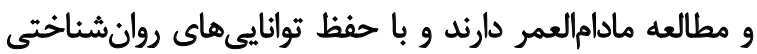

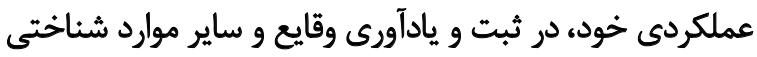

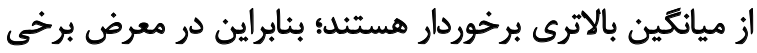

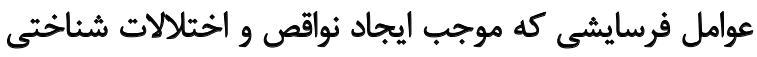

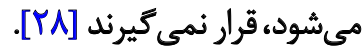

از سوى ديكر مطالعات نشان دادهاند كه برقرارى روابط و

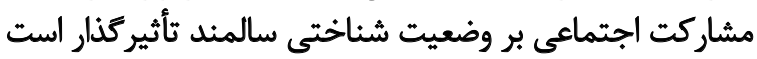

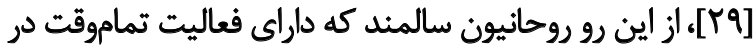

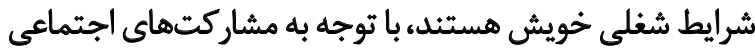

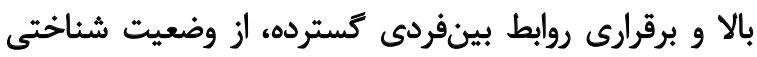

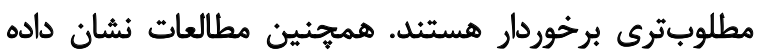

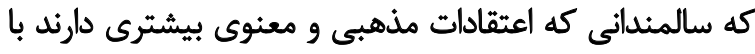

مشكلات شناختى كمترى روبهرو هستئد [• مَّ].

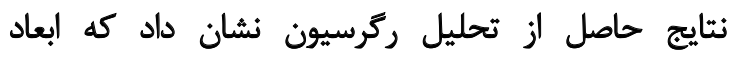

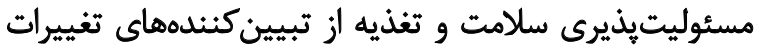

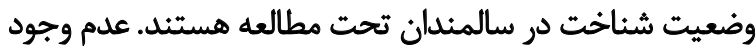

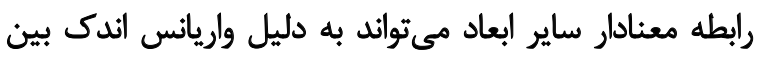
مشاركت كنيدًان در اين ابعاد باشد.

در تبيين ارتباط مسئوليتيذيرى سلامت و شناخت مى تونان

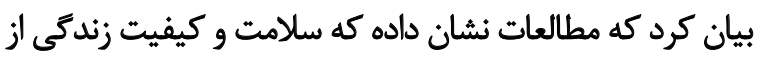

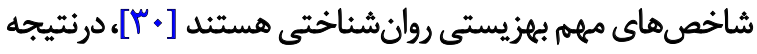

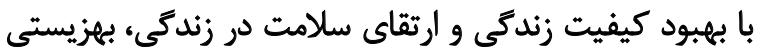

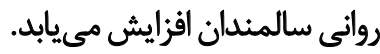

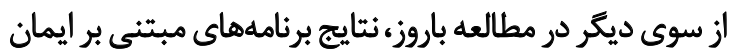

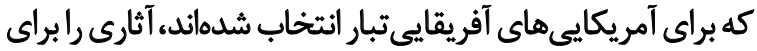

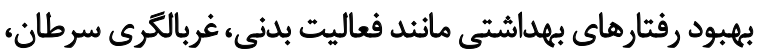

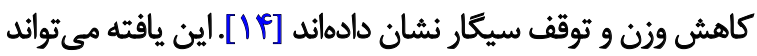

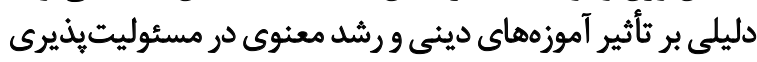

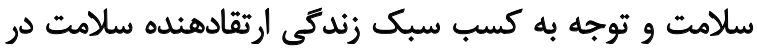
روحانيون سالمند باشد.

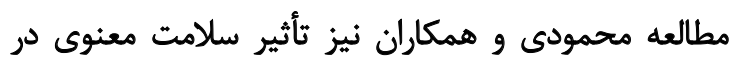

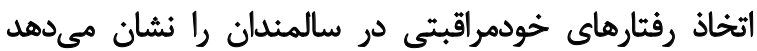

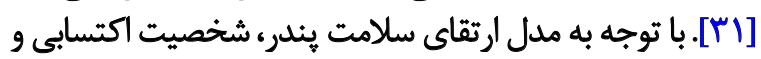

تغذيه و سيس فعاليت فيزيكى بود.

نتايج مطالعه نشان داد كه ميانكين نمره اختلال شناختى در درانيت

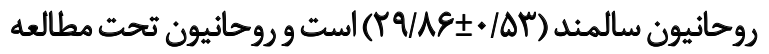

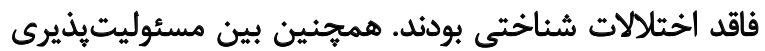

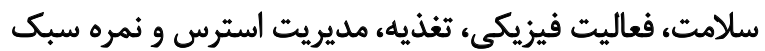

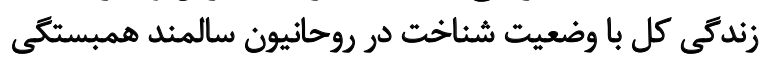
مثبت و معنادار وجود دارد (جدول شماره باخت

با توجه به نتايج ركرسيون خطى جندئانه در مدل مدل نخست،

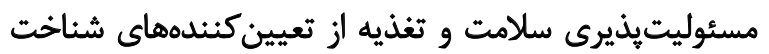

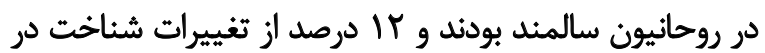

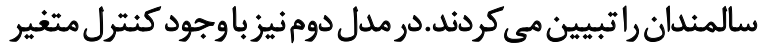

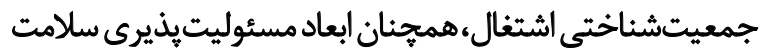

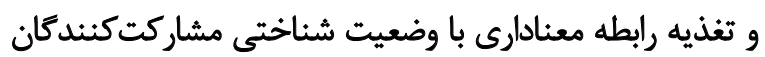

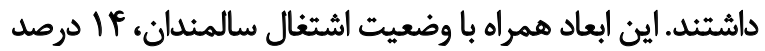

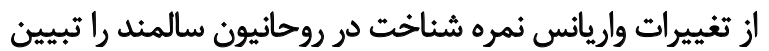

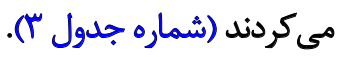

بحث

مطالعه حاضر روى •19 نفر از روحانيون سالمند شهر قم در بر بردي

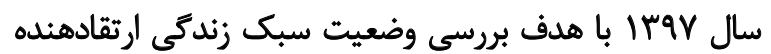

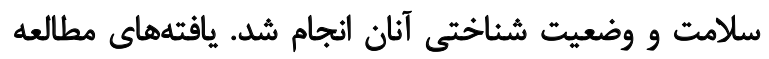

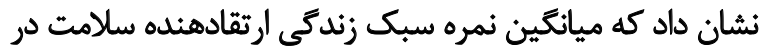

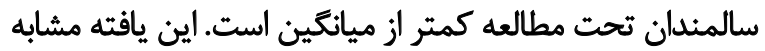

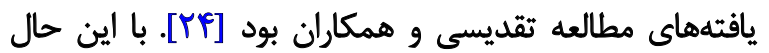

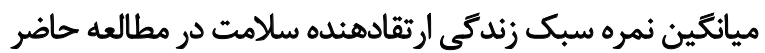

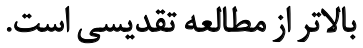

در توجيه اين مطلب ميتوان بيان داشت كه دئ در مطالعه

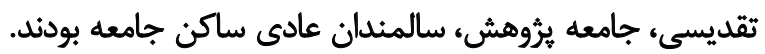

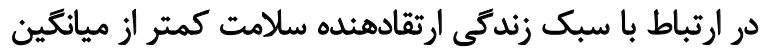

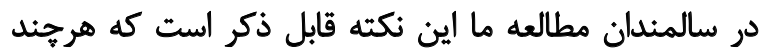

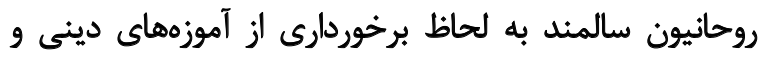

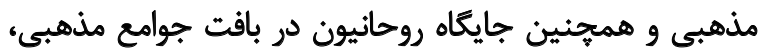

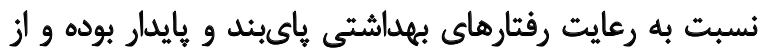

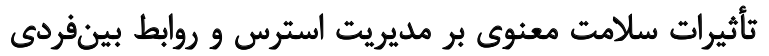

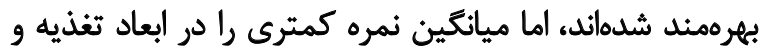

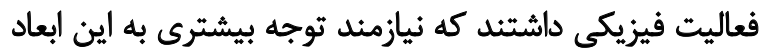

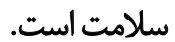
بر ساير مطالعات نيز مشخص شده كه سلامت معنوى و مذهبى

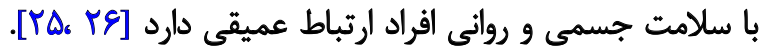

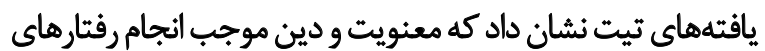

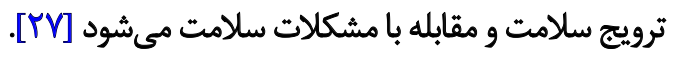
نتايج مطالعه حاضر نشان داد كه روحانيون سالمند تحت 
در روحانيون سالمند به جهت موقعيت مذهبى آنان و مراجعه

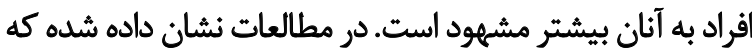

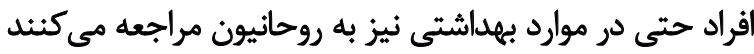

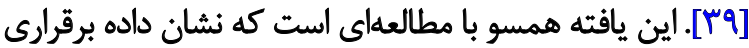

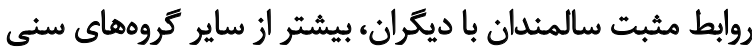

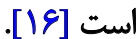

\section{نتيجلَيرى نهايى}

توجه به معنويت و رفتارهاى مرتبط به آن جهت انجام

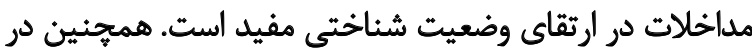

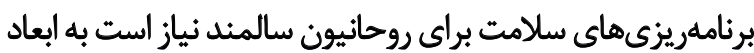

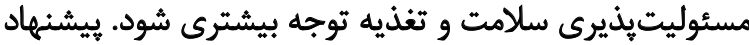

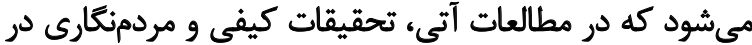

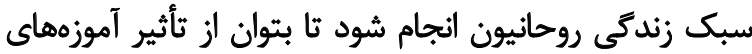

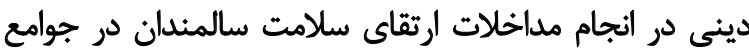
داراى بافت مذهبى بهره كرفت.

از محدوديتهاي اين مطالعه مى توان به نبود منابع و تحقيقات

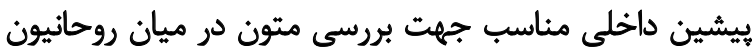

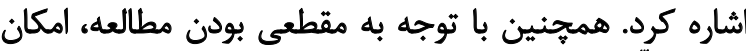

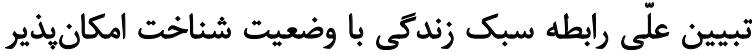

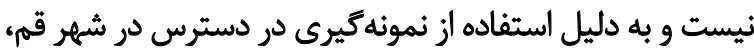

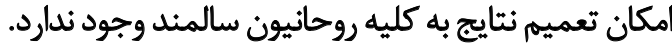
بنابراين يُيشنهاد مىشود در ساير شهرها مطالعاتى در همين

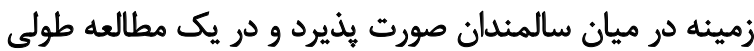

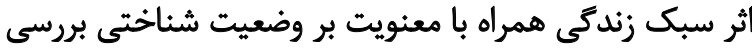

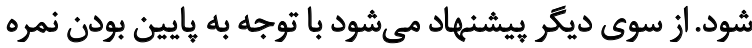

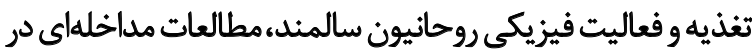
خصوص روشهاى افزيش رفئ رفتارهاى ارتقادهنده سلامت در اين

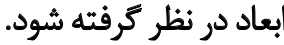

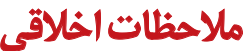

\section{يبروى أز اصول اخلاق يثوهش}

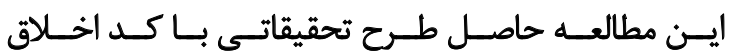

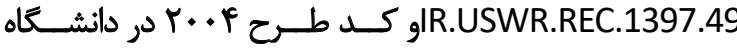

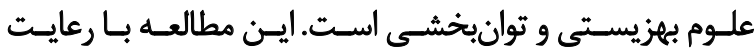

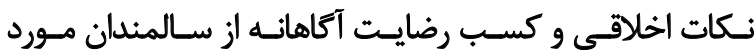

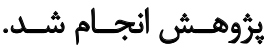

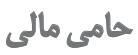

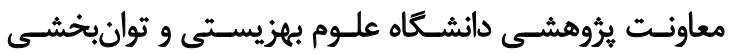

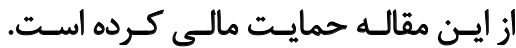

انتسابى، بر باورها، نتيجه و تصويب رفتارهاى ارتقاى سلامت تأثير

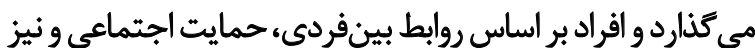

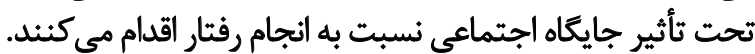

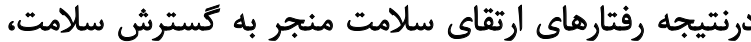

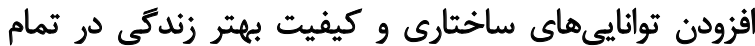

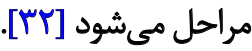

بر همين اساس با توجاه به جايكاه روحانيون در جامعه به بعضى زئل

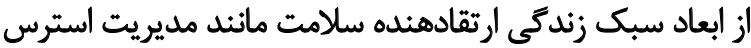

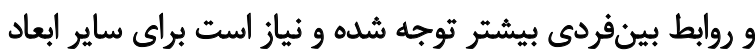

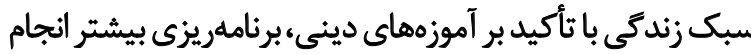

در ارتباط با نقش تبيينكنيده تغذيه در وضعيت شناختى دئي

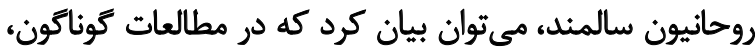

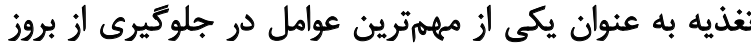

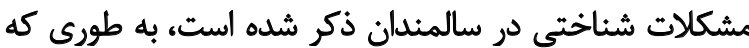

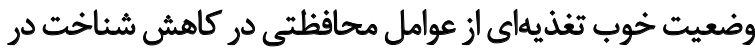

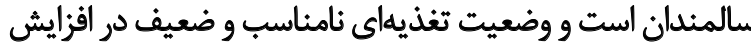

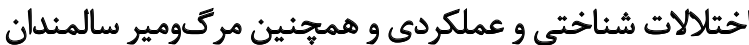

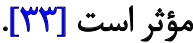
برخى مطالعات به ارتباط بين دين و مذهب با رزيم غذايى افراد

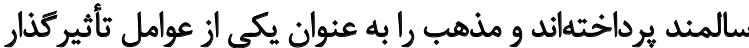

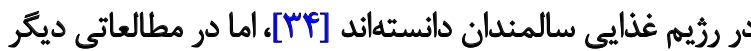

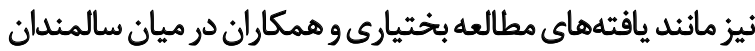

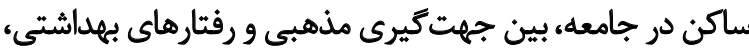

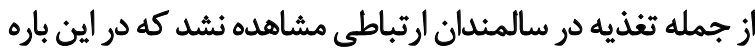

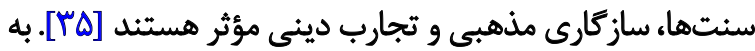

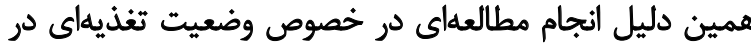
روحانيون سالمند ضرورى به نظر مىرسد. با توجه به نتايج مطالعه، بين سبك زندكى ارتقادهنده سلامت

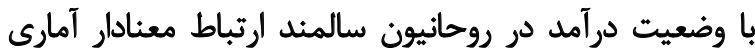
وجود داشت.نتايج مطالعه حاضر با مطالعه ايغار [عَّ] و همكاران، دان.

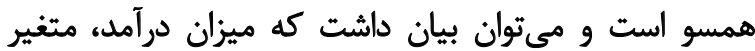

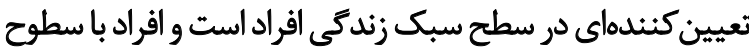

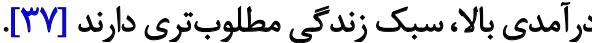
يافتههاى مطالعه حاضر نشان داد كه كمترين ميانكين نمره

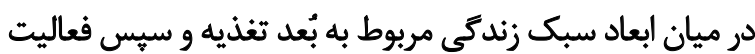

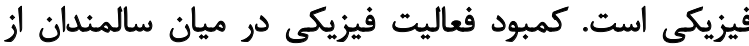

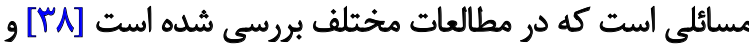

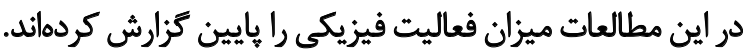

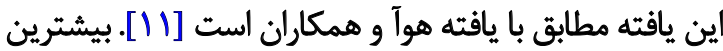

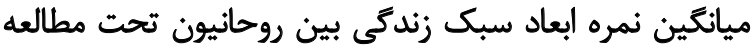
مريوط به بعد مديريت استرس و روابط بين فردى بودين بود. اين مورد 


$$
\text { هشار كث نويسند مكان }
$$

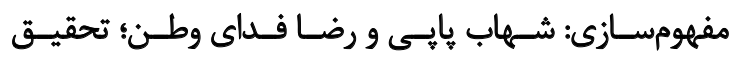

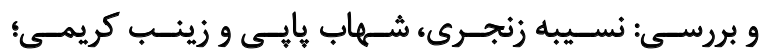

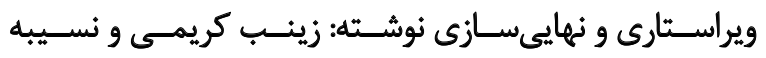

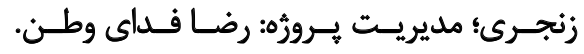

$$
\text { تعارض مناقع }
$$

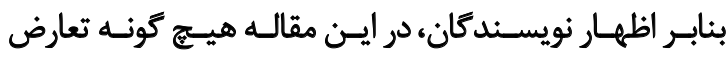

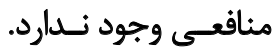

$$
\text { تشكر و قمدرانى }
$$

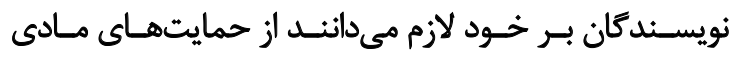

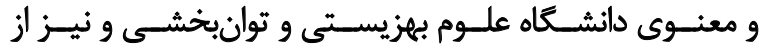

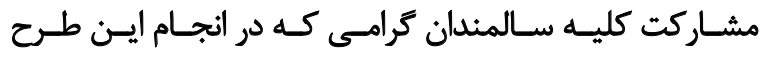

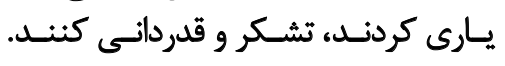




\section{References}

[1] Ren L, Zheng Y, Wu L, Gu Y, He Y, Jiang B, et al. Investigation of the prevalence of cognitive impairment and its risk factors within the elderly population in Shanghai, China. Scientific Reports. 2018; 8(1):3575. [DOI:10.1038/s41598-018-21983-w] [PMID] [PMCID]

[2] Kuo HK, Jones RN, Milberg WP, Tennstedt S, Talbot L, Morris JN, et al. Cognitive function in normal-weight, overweight, and obese older adults: An analysis of the advanced cognitive training for independent and vital elderly cohort. Journal of the American Geriatrics Society. 2006; 54(1):97-103. [DOI:10.1111/j.15325415.2005.00522.x] [PMID] [PMCID]

[3] Park MH. Informant Questionnaire on Cognitive Decline in The Elderly (IQCODE) for classifying cognitive dysfunction as cognitively normal, mild cognitive impairment, and dementia. International Psychogeriatrics. 2017; 29(9):1461-7. [DOI:10.1017/ S1041610217000965] [PMID]

[4] Formiga F, Ferrer A, Chivite D, Alburquerque J, Olmedo C, Mora $\mathrm{JM}$, et al. Predictors of cognitive decline in 85-year-old patients without cognitive impairment at baseline: 2-year follow-up of the Octabaix study. American Journal of Alzheimer's Disease Other Dementia. 2013; 28(2):147-53. [DOI:10.1177/1533317512475021] [PMID]

[5] Taraghi Z, Kamrani A-AA, Foroughan M, Yazdani J, Mahdavi A, Baghernejad SK. Cognitive impairment among elderly patients with chronic heart failure and related factors. Iranian Journal of Psychiatry Behavioral Sciences. 2016; 10(2):e4500. [DOI:10.17795/ ijpbs-4500] [PMID] [PMCID]

[6] Fatehi R, Nezal A, Motalebi A. [Nurses'and elderly's'viewpoints regarding quality of nursing care in the educational hospitals of Sanandaj city (Persian)]. Journal of Urmia Nursing and Midwifery Faculty. 2019; 16(11):779-86. https://www.sid.ir/en/Journal/ ViewPaper.aspx?ID=789944

[7] Stampfer MJ, Hu FB, Manson JE, Rimm EB, Willett WC. Primary prevention of coronary heart disease in women through diet and lifestyle. New England Journal of Medicine. 2000; 343(1):16-22. [DOI:10.1056/NEJM200007063430103] [PMID]

[8] Kurth T, Moore SC, Gaziano JM, Kase CS, Stampfer MJ, Berger $\mathrm{K}$, et al. Healthy lifestyle and the risk of stroke in women. Archives of internal medicine. 2006; 166(13):1403-9. [DOI:10.1001/ archinte.166.13.1403] [PMID]

[9] Hu FB, Manson JE, Stampfer MJ, Colditz G, Liu S, Solomon CG, et al. Diet, lifestyle, and the risk of type 2 diabetes mellitus in women. New England Journal of Medicine. 2001; 345(11):790-7. [DOI:10.1056/NEJMoa010492] [PMID]

[10] Howard EP, Morris JN, Steel K, Strout KA, Fries BE, Moore A, et al. Short-term lifestyle strategies for sustaining cognitive status. BioMed Research International. 2016; 2016:7405748. [DOI:10.1155/2016/7405748] [PMID] [PMCID]

[11] Hua Y, Wang B, Wallen GR, Shao P, Ni C, Hua Q. Health-promoting lifestyles and depression in urban elderly Chinese. PloS One. 2015; 10(3):e0117998. [DOI:10.1371/journal.pone.0117998] [PMID] [PMCID]

[12] Sullivan S, Pyne JM, Cheney AM, Hunt J, Haynes TF, Sullivan $G$. The pew versus the couch: Relationship between mental health and faith communities and lessons learned from a VA/ clergy partnership project. Journal of Religion and Health. 2014; 53(4):1267-82. [DOI:10.1007/s10943-013-9731-0] [PMID]
[13] Zanjari N, Sani MS, Hosseini-Chavoshi M, Rafiey H, Mohammadi-Shahboulaghi F, Citation Zanjari N. Development and validation of successful aging instrument. Iranian Rehabilitation Journal. 2019; 17(2):129-40. [DOI:10.32598/irj.17.2.129]

[14] Baruth M, Wilcox S, Evans R. The health and health behaviors of a sample of African American pastors. Journal of Health Care for the Poor and Underserved. 2014; 25(1):229-41. [DOI:10.1353/ hpu.2014.0041] [PMID] [PMCID]

[15] Bopp M, Baruth M, Peterson JA, Webb BL. Leading their flocks to health? Clergy health and the role of clergy in faith-based health promotion interventions. Family \& Community Health. 2013; 36(3):182-92. [DOI:10.1097/FCH.0b013e31828e671c] [PMID]

[16] Moeini M, Sharifi S, Kajbaf MB. Effect of Islam-based religious program on spiritual wellbeing in elderly with hypertension. 2016; 21(6):566-71. [DOI:10.4103/1735-9066.197683] [PMID] [PMCID]

[17] Moazedi K, Porzoor P, Pirani Z, Adl H, Ahmadi H. The effectiveness of Islamic teaching based religious-spiritual psychotherapy on quality of life, in infertile women (Persian)]. Journal of Health. 2019; 9(5):589-98. [DOI:10.29252/j.health.9.5.589]

[18] Weaver AJ, Koenig HG, Ochberg FM. Posttraumatic stress, mental health professionals, and the clergy: A need for collaboration, training, and research. Journal of Traumatic Stress. 1996 9(4):847-56. [DOI:10.1002/jts.2490090412] [PMID]

[19] Lee H, Park S, Lim K, Lim K, Park Y, Jang J. Association between lifestyle and cognitive impairment among women aged 65 years and over in the Republic of Korea. Educational Gerontology. 2016; 42(3):198-208. [DOI:10.1080/03601277.2015.1085794]

[20] Vaynman S, Gomez-Pinilla F. Revenge of the "sit": how lifestyle impacts neuronal and cognitive health through molecular systems that interface energy metabolism with neuronal plasticity. Journal of Neuroscience Research. 2006; 84(4):699-715. [DOI:10.1002/jnr.20979] [PMID]

[21] Mohammadi Zeidi I, Pakpour Hajiagha A, Mohammadi Zeidi B. [Reliability and validity of Persian version of the health-promoting lifestyle profile (Persian)]. Journal of Mazandaran University of Medical Sciences. 2012; 21(1):102-13. http://jmums. mazums.ac.ir/article-1-955-en.html

[22] Masoumi N, Jafrodi S, Ghanbari A, Ebrahimi S, Kazemnejad E, Shojaee F, et al. [Assessment of cognitive status and related factors in elder people in Rasht (Persian)]. Iranian Journal of Nursing Research.2013; 8(29):80-6. https://www.sid.ir/en/journal/ ViewPaper.aspx?id=318043

[23] Foroughan M, Jafari Z, Shirin Bayan P, Ghaem Magham Farahani Z, Rahgozar M. [Validation of Mini-Mental State Examination (MMSE) in the elderly population of Tehran (Persian)]. Advances in Cognitive Science. 2008; 10(2):29-37. http:/ /icssjournal. ir/article-1-422-en.html

[24] Taghdisi MH, Estebsari F, Rahimi Foroushani A, Eftekhar Ardebili H, Shojaeizadeh D, Dastoorpoor M, et al. [The educational program based on the successful aging approach in elders healthpromoting behaviors: A clinical trial study (Persian)]. Razi Journal of Medical Sciences. 2014; 21(125):26-36._http://rims.iums ac.ir/article-1-3420-en.html

[25] Gonzalez P, Castañeda SF, Dale J, Medeiros EA, Buelna C, Nuñez A, et al. Spiritual well-being and depressive symptoms among cancer survivors. Supportive Care in Cancer. 2014; 22(9):2393-400. [DOI:10.1007/s00520-014-2207-2] [PMID] [PMCID] 
[26] Bonelli RM, Koenig HG. Mental disorders, religion and spirituality 1990 to 2010: A systematic evidence-based review. Journal of Religion and Health. 2013; 52(2):657-73. [DOI:10.1007/s10943-013-9691-4] [PMID]

[27] Tate JD. The role of spirituality in the breast cancer experiences of African American women. Journal of Holistic Nursing. 2011; 29(4):249-55. [DOI:10.1177/0898010111398655] [PMID]

[28] Jutkowitz E, MacLehose RF, Gaugler JE, Dowd B, Kuntz KM, Kane RL. Risk factors associated with cognitive, function$\mathrm{al}$, and behavioral trajectories of newly diagnosed dementia patients. Journals of Gerontology Series A: Biomedical Sciences Medical Sciences. 2016; 72(2):251-8. [DOI:10.1093/gerona/ glw079] [PMID] [PMCID]

[29] Shahbazi MR, Foroughan M, Salman Roghani R, Rahgozar M. [The relationship between disability and variables of depression, cognitive status, and morale among older people (Persian)]. Iranian Journal of Ageing. 2016; 11(1):132-41. [DOI:10.21859/sija-1101132]

[30] Corsentino EA, Collins N, Sachs-Ericsson N, Blazer DG. Religious attendance reduces cognitive decline among older women with high levels of depressive symptoms. Journals of Gerontology Series A: Biomedical Sciences Medical Sciences. 2009; 64(12):1283-9. [DOI:10.1093/gerona/glp116] [PMID] [PMCID]

[31] Mohammadi M, Alavi M, Bahrami M, Zandieh Z. Assessment of the relationship between spiritual and social health and the self-care ability of elderly people referred to community health centers. Iranian Journal of Nursing and Midwifery Research. 2017; 22(6):471-5. [DOI:10.4103/ijnmr.IJNMR_171_16] [PMID] [PMCID]

[32] Pender NJ. Health promotion model manual [Internet]. 2011 [Updated 2011]. Available from: https://deepblue.lib.umich. edu/bitstream/handle/2027.42/85350/HEALTH_PROMOTION_MANUAL_Rev_5-2011.pdf?sequence=1

[33] Zanetti M, Cappellari GG, Ratti C, Ceschia G, Murena L, De Colle $\mathrm{P}$, et al. Poor nutritional status but not cognitive or functional impairment per se independently predict 1 year mortality in elderly patients with hip-fracture. Clinical Nutrition. 2019; 38(4):1607-12. [DOI:10.1016/j.clnu.2018.08.030] [PMID]

[34] Hye-Cheon Kim K, Alex Mcintosh W, Kubena KS, Sobal J. Religion, social support, food-related social support, diet, nutrition, and anthropometrics in older adults. Ecology of Food Nutrition. 2008; 47(3):205-28. [DOI:10.1080/03670240802035068]

[35] Bakhtiari A, Yadollahpur M, Omidvar S, Ghorbannejad S, Bakouei F. Does religion predict health-promoting behaviors in community-dwelling elderly people? Journal of Religion Health. 2019; 58(2):452-64. [DOI:10.1007/s10943-018-0710-3] [PMID]

[36] Aygar H, Akbulut Zencirci S, Öztürk Emiral G, Alaiye M SA, Önsüz MF, Işıklı B, et al. Assessment of health promoting lifestyle behaviours of adults living in the semi-rural area. Northern Clinics of İstanbul. 2019; 6(1):13-20. [DOI:10.14744/ nci.2017.19327] [PMID] [PMCID]

[37] Baumann M, Le Bihan E, Chau K, Chau N. Associations between quality of life and socioeconomic factors, functional impairments and dissatisfaction with received information and home-care services among survivors living at home two years after stroke onset. BMC neurology. 2014; 14:92. [DOI:10.1186/1471-2377-14-92] [PMID] [PMCID]
[38] Papi S, Ramezani T, Aalipour R, Naderiyan S, Fadayevatan R, Nazarpour A. [Assessment of physical activity status and its effective factors in elderly people of Khorramabad city (Persian)]. Health and Development Journal. 2019; 8(3):280-91. [DOI: $10.22034 / 8.3 .280]$

[39] Weaver AJ, Larson DB, Flannelly KJ, Stapleton CL, Koenig HG. Mental health issues among clergy and other religious professionals: A review of research. Journal of Pastoral Care \& Counseling. 2002; 56(4):393-403. [DOI:10.1177/154230500205600408] [PMID] 